\title{
Analyzing the surface properties of acrylic-based water-soluble polymers
}

\author{
Demet Topaloğlu Yazııı ${ }^{a}$, Hayrullah Çetinkaya ${ }^{b}$, Musa Şölener ${ }^{a}$, and Adile Albayrak ${ }^{a}$ \\ aDepartment of Chemical Engineering, Eskişehir Osmangazi University, Eskişehir, Turkey; ${ }^{b}$ Department of Chemical \\ Engineering, Izmir Institute of Technology, Izmir, Turkey
}

\begin{abstract}
The surface properties of newly synthesized $N$-methoxy isopropyl acrylamide, $N$-methoxy isopropyl methacrylamide, cyclo propyl acrylamide, and cyclo propyl methacrylamide polymers were investigated using inverse gas chromatography. The highest dispersive component of the surface energy value was obtained for cyclo propyl methacrylamide at $30^{\circ} \mathrm{C}$. The values obtained for all polymers were decreasing with the increasing temperature. The values obtained for the acidic and the basic parameters revealed strong basic characters for the surface of $\mathrm{N}$-methoxy isopropyl methacrylamide and cyclo propyl methacrylamide polymers and weak basic characters for the surface of $\mathrm{N}$-methoxy isopropyl acrylamide and cyclo propyl acrylamide polymers.
\end{abstract}

\section{ARTICLE HISTORY}

Submitted 8 April 2017

Accepted 1 May 2017

\section{KEYWORDS}

Acrylamide polymers; inverse gas chromatography; surface acid-base properties; surface energy

\section{Introduction}

The polymers are being called as intelligent, environmentally sensitive, and stimuli-sensitive responds according to an external stimulus like ionic strength, solvent type, temperature, $\mathrm{pH}$, and electric or magnetic field. The physical responds may be as precipitation, solution, swelling, or shrinking and so on. For instance, one component of the temperature-sensitive polymers should be soluble depending on the temperature in solvent which is generally water. When the temperature reaches a critical value, the polymer phase changes and the phase separation can be observed. This temperature is called as lower critical solution temperature (LCST). In general, temperature-sensitive polymers are synthesized by polymerization of the monomers in the form of $\mathrm{N}$-alkyl acrylamide. In this study, the synthesized polymers are; $N$-methoxy isopropyl acrylamide (NMIPA), $N$-methoxy isopropyl methacrylamide (NMIPMA), cyclo propyl acrylamide (CPA), and cyclo propyl methacrylamide (CPMA). The LCST value for $N$-methoxy isopropyl acrylamide polymer (PNMIPA) is of $35-45^{\circ} \mathrm{C}^{[1]}$ and for cyclo propyl acrylamide polymer (PCPA) is of $38-54.5^{\circ} \mathrm{C} .{ }^{[2]}$ The LCST value for PNIPMA is of $45-50^{\circ} \mathrm{C}$ and for cyclo propyl methacrylamide polymer (PCPMA) is of $45-55^{\circ} \mathrm{C}$. The LCST values of the last two polymers were determined in this study and to the best of our knowledge there have been no researches of these polymers. The LCST value decreases linearly with increasing polymer concentration for all studied polymers. Furthermore, they are molecularly soluble in water and organic solvents like tetrahydrofuran (THF) and dichloromethane (DCM) at room temperature.

Water-soluble polymers have a wide range of industrial applications like food, pharmaceuticals, paint, textiles, paper, constructions, adhesives, coatings, and water treatment. The polymers have been synthesized to suit specific needs or rather to solve specific problems. ${ }^{[3]}$ Likewise, the surface properties of the polymer surfaces should meet the particular requirements of these applications.

There are a lot of methods available for investigating the surfaces of polymers but inverse gas chromatography (IGC) has been shown ${ }^{[4-6]}$ to be useful for this type of work. It is a reliable and faster

CONTACT Demet Topaloğlu Yazıcı @emett@ogu.edu.tr $\mathrm{Q}$ Department of Chemical Engineering, Faculty of Engineering and Architecture, Eskişehir Osmangazi University, Eskişehir 26480, Turkey. 
method according to the other conventional methods. IGC is known to be the reverse of the conventional gas chromatography in which a nonvolatile material to be investigated is immobilized in a column. Small amounts of 'probe' solvents are injected into a flow of carrier gas over the material under investigation and the retention time depends on the interactions between the probe and the surface of nonvolatile material. Using probes with a range of properties, the nature of the surface can be defined with good accuracy. ${ }^{[6]}$ Some of these properties are; the adsorption thermodynamic parameters, acid-base characteristics, solubility parameters, diffusion coefficients, interfacial energy, surface tension, and surface area.

In this study, the surface properties of newly synthesized water-soluble polymers were determined by IGC at infinite dilution. The determined surface properties like the dispersive component of the surface energy and the acidity and basicity of the polymer surfaces gave some insights about the surfaces. Besides these, the adsorption enthalpies of the probes $\left(\Delta H^{\circ}\right)$ on polymers were also calculated.

\section{Experimental}

The polymers used in this study were synthesized using solution polymerization. Polymerizations were done by solving appropriate amounts of azobisisobutyronitrile with ethanol and then monomers were added. Polymerization media was purged with nitrogen for $30 \mathrm{~min}$ and polymerization was continued for $12 \mathrm{~h}$ at $70^{\circ} \mathrm{C}$. After that, the solvent was removed. ${ }^{[1]}$ The polymers can be separated by precipitating in basic solution. The obtained polymers were dissolved in very small amount of DCM and crystallized with diethyl ether. The dissolution and crystallization process were repeated several times. The synthesized polymers were; NMIPA, NMIPMA, CPA, and CPMA polymers. The molecular formulas of the synthesized polymers are given in Table 1. The molecular weights were arranged around $3,000 \mathrm{~g} \mathrm{~mol}^{-1}$ while synthesizing polymers. The values were as follows: PCPMA $2,700 \mathrm{~g} \mathrm{~mol}^{-1}$, PCPA $3,200 \mathrm{~g} \mathrm{~mol}^{-1}, N$-methoxy isopropyl methacrylamide polymer (PNMIPMA) $3,700 \mathrm{~g} \mathrm{~mol}^{-1}$, and PNMIPA $3,100 \mathrm{~g} \mathrm{~mol}^{-1}$. The molecular weights of polymers were determined by gel permeation chromatography.

To investigate the polymers by IGC, the soaking method of Al-Saigh and Munk ${ }^{[7]}$ was used to coat the polymers on Chromosorb W-AW DMCS (60-80 mesh; Alltech). Standard ashing procedures ${ }^{[5]}$ showed the weight of coated PNMIPA, PNMIPMA, PCPA, and PCPMA as 9.8, 10.2, 10.0, and $9.6 \%$ of the total solids, respectively. The coated materials were packed into stainless steel column

Table 1. The molecular formulas of the synthesized polymers.

Monomer


of IGC. The nonpolar adsorbates (probes) used were $n$-hexane, $n$-heptane, $n$-octane, $n$-nonane, (Merck, reagent grade). The polar probes used were DCM, trichloromethane (TCM), ethyl acetate (EA), THF, and diethyl ether which are characterized by their donor and acceptor numbers. The donor numbers $(\mathrm{DN})$ and modified acceptor numbers $\left(\mathrm{AN}^{*}\right)$ were taken. ${ }^{[4,8-10]}$ The chromatographic experiments were performed with Agilent 7890 gas chromatograph equipped with a flame ionization detector. A stainless steel column (2-m long and 5.35-mm ID) previously washed with methanol and acetone was packed with about $2 \mathrm{~g}$ for each material. The adsorbents were conditioned at the highest studied temperatures in the nitrogen gas flow for $24 \mathrm{~h}$ before the measurements. Retention times for each probe were measured below the glass transition temperatures $\left(T_{\mathrm{g}}\right)$ [The $T_{\mathrm{g}}$ values of the PNMIPA, PNMIPMA, PCPA, and PCPMA were determined as $130,122,46$, and $42^{\circ} \mathrm{C}$, respectively by Perkin Elmer/Pyris 1-Differential Scanning Calorimeter in the laboratories of the TÜBİTAK Research Institute (Scientific and Technical Research Council of Turkey)]. Measuring below $T_{\mathrm{g}}$ is assumed that surface effects predominate sufficiently to neglect contributions from bulk sorption. ${ }^{[4,11]}$ Symmetrical peaks were recorded and the retention time was taken as the peak maximum. Elution times were found to be independent from the vapor quantity injected (Henry's Law region). Retention times were obtained from differences between peak maxima and methane markers. ${ }^{[12]}$

\section{Results and discussion}

Inverse gas chromatography is generally performed at infinite dilution, so that the probe-probe interactions are negligible, and the retention time is governed by solid-probe interactions only. ${ }^{[1,12]}$ The net retention volume $\left(V_{\mathrm{n}}\right)$ is the amount of carrier gas required to elute the injected probe gas from the column and is calculated mainly from retention time as described in the following equation $^{[5]}$ :

$$
V_{\mathrm{n}}=\left(t_{\mathrm{r}}-t_{\mathrm{m}}\right) \cdot F_{\mathrm{r}} \cdot \frac{T_{\mathrm{c}}}{T_{\mathrm{r}}} \cdot\left(\frac{P_{\mathrm{o}}-P_{\mathrm{w}}}{P_{\mathrm{o}}}\right) \cdot j
$$

where $t_{\mathrm{r}}$ is the retention time of probe, $t_{\mathrm{m}}$ is the retention time of methane, $F_{\mathrm{r}}$ is volumetric flow rate at column outlet and at room temperature $T_{\mathrm{r}}(\mathrm{K}), T_{\mathrm{c}}$ is the column temperature $(\mathrm{K}), P_{\mathrm{o}}$ is the column outlet pressure, $P_{\mathrm{w}}$ is the vapor pressure of water at $T_{\mathrm{r}}$, and $j$ is James-Martin gas compressibility correction factor.

Once calculating the net retention volume, it provides a way to obtain the other physicochemical parameters of the surface such the dispersive component of the surface energy $\left(\gamma_{s}^{d}\right)$ and the acid-base interactions of the stationary phase in the column. $\gamma_{S}^{\mathrm{d}}$ is attributed to the solid's potential for London-type interactions and can be determined by IGC using the nonpolar probes (alkanes). We begin with determining the dispersive component of surface energy using the retention time. ${ }^{[4,8]}$

$$
\mathrm{RT}_{\mathrm{c}} \ln V_{\mathrm{n}}=2 N \cdot\left(\gamma_{\mathrm{S}}^{\mathrm{d}}\right)^{1 / 2} \cdot a \cdot\left(\gamma_{\mathrm{L}}^{\mathrm{d}}\right)^{1 / 2}+\text { constant }
$$

where $\gamma_{\mathrm{L}}^{\mathrm{d}}$ is the liquid tension of the probe molecule, $a$ is its cross-sectional area, ${ }^{[8,9]}$ and $N$ is the Avagadro number. The slope of the plot of $a \cdot\left(\gamma_{\mathrm{L}}^{\mathrm{d}}\right)^{1 / 2}$ versus $\mathrm{RT}_{\mathrm{c}} \ln V_{\mathrm{n}}$ gives the dispersive component of the surface energy of studied polymer.

The obtained plots for polymers are given in Figure 1. Linear correlations were obtained for the $n$-alkane series. The $\gamma_{s}^{\mathrm{d}}$ values calculated from the slope of these plots are given in Tables 2 and 3. It was observed that the values decreased with temperature as expected. The $\gamma_{S}^{\mathrm{d}}$ values obtained for polymers at 30 and $40^{\circ} \mathrm{C}$ decrease in the following order: PCPMA $>$ PNMIPA $>$ PCPA $>$ PNMIPMA. This order could not be correlated with either the cyclic structure or the different functional group. However, the order is the opposite of the sequence of molecular weights of polymers (PNMIPMA $>$ PCPA $>$ PNMIPA $>$ PCPMA), the insignificant variation between the molecular weights may not thought to be the main reason. Accordingly, the effect of the molecular weights of the polymers on 


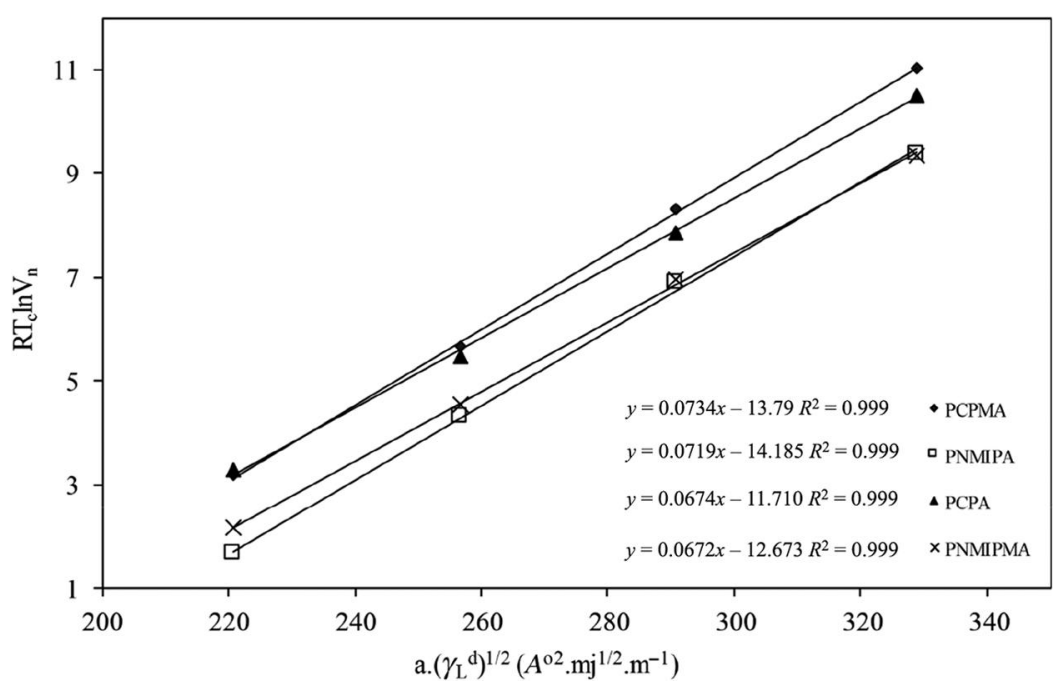

Figure 1. The plot of $a \cdot\left(\gamma_{\mathrm{L}}^{\mathrm{d}}\right)^{1 / 2}$ versus $\mathrm{RT} \mathrm{c} \ln V_{\mathrm{n}}$ for NMIPA, NMIPMA, CPA, and CPMA polymers at studied temperatures.

Table 2. $\quad \gamma_{s}^{d}$ values for NMIPA and NMIPMA polymers at studied temperatures.

\begin{tabular}{lcc}
\hline & \multicolumn{2}{c}{$\gamma_{\mathrm{S}}^{\mathrm{d}}\left(\mathrm{mJ} \mathrm{m}^{-2}\right)$} \\
\cline { 2 - 3 }$T\left({ }^{\circ} \mathrm{C}\right)$ & NMIPA & NMIPMA \\
\hline 30 & 35.71 & 31.13 \\
35 & 35.05 & 30.00 \\
40 & 34.07 & 27.31 \\
\hline
\end{tabular}

the surface energies was discussed. ${ }^{[13,14]}$ Furthermore, it may be explained by the physical factors like structural heterogeneities of molecular size. It is very well known that the dispersive parameters are very sensitive to the differences between the surfaces of chemically similar polymers and the change occurring on such surfaces under the influence of various physical factors. ${ }^{[15]}$

The usage of water-soluble polymers especially for human health subjects, like pharmaceuticals and nutrition, requires the knowledge of the surface acidity/basicity or the acid/base surface interactions. IGC method is also used to examine the surface acidity/basicity of the investigated material. To examine the acidity/basicity; the specific component free energy of adsorption, $\Delta G^{\mathrm{sp}}$ and the specific enthalpy, $\Delta H^{\text {sp }}$ should be determined as follows ${ }^{[4]}$ :

A plot ( $n$-alkane plot) was drawn above while determining the $\gamma_{S}^{\mathrm{d}}$ from the slope of the linear plot according to Equation (2). Polar probes were also placed in this figure. The difference between the point of polar probe in the figure and the linear plot gives $\Delta G^{\mathrm{sp}}$ value:

$$
-\Delta G^{\mathrm{sp}}=-\left(\Delta G^{\mathrm{o}}-\Delta G^{\mathrm{d}}\right)=\mathrm{RT}_{\mathrm{c}} \ln \left(V_{\mathrm{n}} / V_{\mathrm{n}, \mathrm{ref}}\right)
$$

Table 3. $\gamma_{s}^{\mathrm{d}}$ values for CPA and CPMA polymers at studied temperatures.

\begin{tabular}{lcc}
\hline & \multicolumn{2}{c}{$\gamma_{\mathrm{S}}^{\mathrm{d}}\left(\mathrm{mJ} \mathrm{m}^{-2}\right)$} \\
\cline { 2 - 3 }$T\left({ }^{\circ} \mathrm{C}\right)$ & $\mathrm{CPA}$ & $\mathrm{CPMA}$ \\
\hline 30 & 31.35 & 37.13 \\
40 & 30.09 & 34.13 \\
50 & 28.99 & 33.18 \\
60 & 27.55 & 32.23 \\
\hline
\end{tabular}


where $V_{\mathrm{n}}$ is the net retention volume of the polar probe and $V_{\mathrm{n} \text {,ref }}$ is the net retention volume of a reference $n$-alkane. The free energy of adsorption for any probe, $\Delta G^{\mathrm{o}}$ is the sum of nonpolar and polar interactions.

After calculating the $\Delta G^{\text {sp }}$ values, the specific enthalpy, $\Delta H^{\mathrm{sp}}$ can be found from the well-known equation by finding the slope of the straight line;

$$
\frac{\Delta G^{\mathrm{sp}}}{T}=\frac{\Delta H^{\mathrm{sp}}}{T}-\Delta S^{\mathrm{sp}}
$$

$\Delta H^{\mathrm{sp}}$ values were related to acid-base properties with the following equation:

$$
-\frac{\Delta H^{\mathrm{sp}}}{\mathrm{AN}^{*}}=K_{\mathrm{A}} \frac{\mathrm{DN}}{\mathrm{AN}^{*}}+K_{\mathrm{D}}
$$

where $K_{\mathrm{A}}$ and $K_{\mathrm{D}}$ denote the acceptor constant and the donor constant of the stationary phase, respectively. $\mathrm{DN}$ and $\mathrm{AN}^{*}$ are donor and modified acceptor numbers, respectively, corresponding to the polar probes. ${ }^{[15]}$ According to Equation (5), the plot of $-\Delta H^{\mathrm{sp}} / \mathrm{AN}^{*}$ versus DN/AN* can be used to determine the $K_{\mathrm{A}}$ and $K_{\mathrm{D}}$ from the slope and intercept of the straight line (Figure 2).

In general, the $K_{\mathrm{D}} / K_{\mathrm{A}}$ ratio is used to evaluate whether the polymer is acidic or basic. If this ratio is bigger than 1, the surface is considered as basic and if the ratio is smaller than 1, it is considered as acidic. The obtained $K_{\mathrm{A}}, K_{\mathrm{D}}$, and $K_{\mathrm{D}} / K_{\mathrm{A}}$ values are given in Table 4 . DCM, TCM, ethyl acetate, and THF polar probes were used for PCPMA and PNMIPMA. DCM, ethyl acetate, and THF polar probes were used for PNMIPA and DCM, TCM, diethyl ether, and THF polar probes were used for PCPA to provide high regression coefficients.

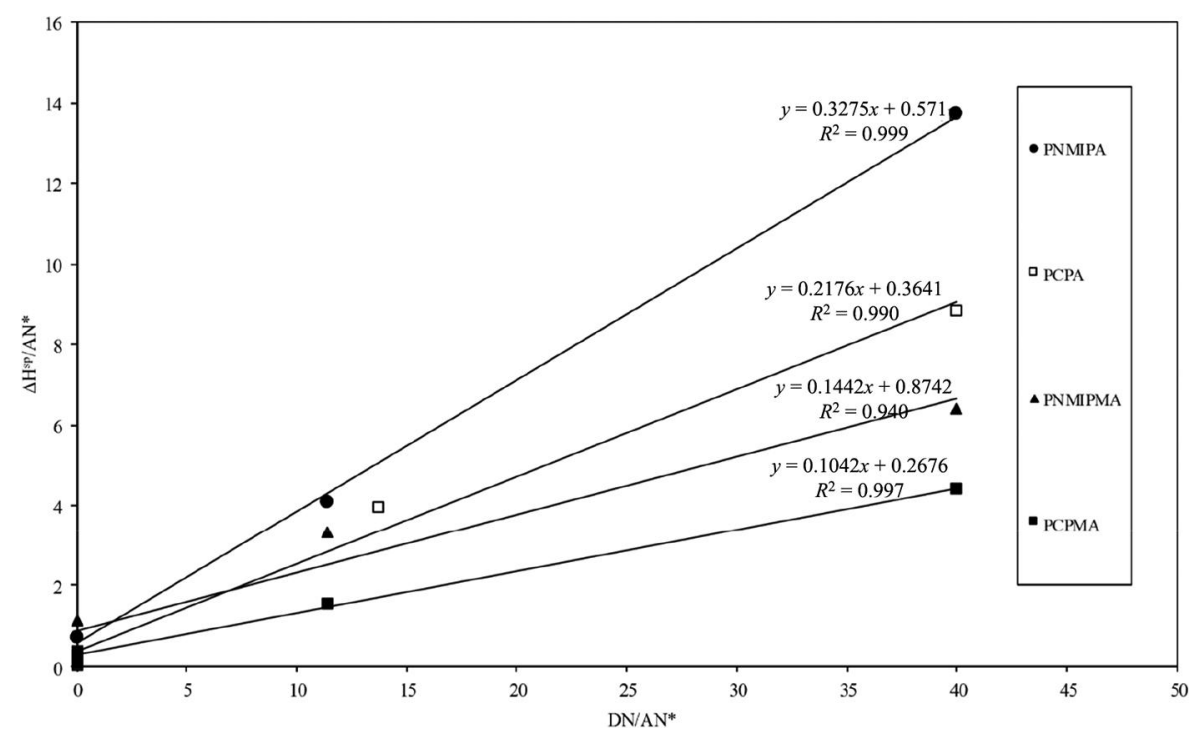

Figure 2. The plot of $\Delta H^{\mathrm{sP}} / \mathrm{AN}^{*}$ versus $\mathrm{DN} / \mathrm{AN}^{*}$ for NMIPA, NMIPMA, CPA, and CPMA polymers.

Table 4. $K_{\mathrm{A}}, K_{\mathrm{D}}$, and $K_{\mathrm{D}} / K_{\mathrm{A}}$ values for all polymers.

\begin{tabular}{lcccr}
\hline & NMIPMA & CPMA & NMIPA & CPA \\
\hline$K_{\mathrm{A}}$ & 0.14 & 0.10 & 0.33 & 0.22 \\
$K_{\mathrm{D}}$ & 0.87 & 0.27 & 0.57 & 0.36 \\
$K_{\mathrm{D}} / K_{\mathrm{A}}$ & 6.06 & 2.57 & 1.74 & 1.67 \\
\hline
\end{tabular}


Table 5. Adsorption enthalpy of the probes on polymers.

\begin{tabular}{lcccr}
\hline & \multicolumn{3}{c}{$-\Delta \mathrm{H}^{\circ}\left(\mathrm{kJ} \mathrm{mol}^{-1}\right)$} \\
\cline { 2 - 5 } Probes & NMIPA & NMIPMA & CPA & CPMA \\
\hline$n$-Hexane & 26.36 & 18.84 & 25.76 & 32.04 \\
$n$-Heptane & 31.87 & 27.66 & 27.46 & 35.48 \\
$n$-Octane & 39.55 & 36.03 & 29.99 & 39.06 \\
$n$-Nonane & 46.27 & 46.42 & 37.04 & 45.35 \\
DCM & 44.20 & 39.64 & 25.21 & 30.06 \\
TCM & 24.74 & 20.10 & 32.64 & 19.93 \\
THF & 59.68 & 27.88 & 47.50 & 33.92 \\
EA & 57.78 & 44.13 & - & 36.74 \\
\hline
\end{tabular}

The surfaces of all studied polymers exhibited basic character according to the $K_{\mathrm{D}} / K_{\mathrm{A}}$ ratios. NMIPMA polymer is found to be the strongest basic polymer. The $K_{\mathrm{D}} / K_{\mathrm{A}}$ ratios of the methacrylamide polymers (PNMIPMA and PCPMA) were higher than the acrylamide polymers (PNMIPA and PCPA). The NMIPMA and NMIPA polymers showed higher basicity due to the cyclo-structured polymers.

The adsorption enthalpy has important signs about the interactions of the adsorbed molecule and the adsorbent. An adsorption process on a polymer depends on some properties like the molecular size and the polarity of adsorbed molecule, the structure, porosity, surface area of the polymer, and the adsorption temperature. At infinite dilution, $\Delta H^{0}$ can be determined with the differential adsorption heat of the probe.

$$
\Delta H^{0}=-R \frac{d \ln V_{\mathrm{n}}}{d\left(1 / T_{\mathrm{c}}\right)}
$$

The values of $-\Delta H^{0}$ (Table 5) increase with increasing carbon number and linear increments were obtained for the $n$-alkanes. The increment with increasing carbon number is expected in homolog series. Polar probes were chosen according to their acidity/basicity characters. As can be seen from the table, almost similar retention mechanisms were observed for polar probes. Polar probes which are grouped as acid (TCM, DCM) had smaller adsorption enthalpies than the basic probes (THF, EA).

\section{Conclusion}

The surface properties like adsorption enthalpy, the dispersive component of surface energy, and the Lewis acid-base parameters of newly synthesized NMIPA, NMIPMA, CPA, and CPMA polymers were studied by IGC. This method performed at infinite dilution is successfully used to investigate the surface of polymers for years. The $\gamma_{S}^{\mathrm{d}}$ values are attributed to the wettability and adhesive characters of materials. In this study, PCPMA has the highest and PNMIPMA has the lowest $\gamma_{\mathrm{S}}^{\mathrm{d}}$ values at studied temperatures. The obtained $K_{\mathrm{A}}$ and $K_{\mathrm{D}}$ values showed that NMIPMA polymer is the strongest basic polymer.

\section{References}

[1] Uguzdogan, E., and O. S. Kabasakal. 2010. Synthesis and characterization of thermally-sensitive polymer: Poly (aminomethoxypropylacrylamide). Colloids Surf. A Physicochem. Eng. Aspects 368:129-136.

[2] Uguzdogan, E., E. B. Denkbas, and O. S. Kabasakal. 2013. Investigation of temperature sensitivity behaviors of water soluble polyacrylamides. J. Appl. Polym. Sci. 127(6):4374-4384.

[3] Kadajji, V. G., and G. V. Betageri. 2011. Water soluble polymers for pharmaceutical applications. Polymers 3:1972-2009.

[4] Aşkın, A., and D. T. Yazıcı. 2008. A study of the surface analysis of some water-soluble polymers by inverse gas chromatography. Surf. Interface Anal. 40:1237-1241.

[5] Conder, J. R., and C. L. Young. 1979. Physicochemical Measurement by Gas Chromatography. New York: Wiley-Interscience. 
[6] Ansari, D. M., and G. J. Price. 2004. Chromatographic estimation of filler surface energies and correlation with photodegradation of kaolin filled polyethylene. Polymer. 45(6):1823-1831.

[7] Al-Saigh, Y. Z., and P. Munk. 1984. Study of polymer-polymer interaction coefficient in polymer blends using inverse gas chromatography. Macromolecules 1:803-809.

[8] Schultz, J., L. Lavielle, and C. Martin. 1987. Surface properties of carbon fibers determined by inverse gas chromatography. J. Adhesion 23:45-60.

[9] Kamdem, D. P., S. K. Bose, and P. Luner. 1993. Inverse gas chromatography characterization of birch wood meal. Langmuir 9:3039-3044.

[10] Panzer, U., and H. P. Schreiber. 1992. On the evaluation of surface interactions by inverse gas chromatography. Macromolecules 25:3633-3637.

[11] Mukhopadhyay, P., and H. P. Schreiber. 1993. Inverse gas chromatography for polymer surface characterization above and below $T_{\mathrm{g}}$. Macromolecules 26:6391-6396.

[12] Topaloğlu Yazıcı, D. 2010. Investigating the surface properties of polymer-coated clay by IGC. Surf. Interface Anal. 42:974-977.

[13] Kusy, R. P., and M. J. Katz. 1976. Effect of molecular weight on the fracture surface energy of poly(methyl methacrylate) in cleavage. J. Mater. Sci. 11:1475-1486.

[14] Moreira, J. C., and N. R. Demarquette. 2001. Influence of temperature, molecular weight, and molecular weight dispersity on the surface tension of PS, PP, and PE. I. Experimental. J. Appl. Polym. Sci. 82:1907-1920.

[15] Voelkel, A., E. Andrzejewska, R. Maga, and M. Andrzejewski. 1999. Examination of surfaces of solid polymers by inverse gas chromotography: 1. Dispersive properties. Polymer 37(3):455-462. 